\title{
Observation Start Day
}

National Cancer Institute

\section{Source}

National Cancer Institute. Observation Start Day. NCI Thesaurus. Code C82570.

The first day of an observation. 